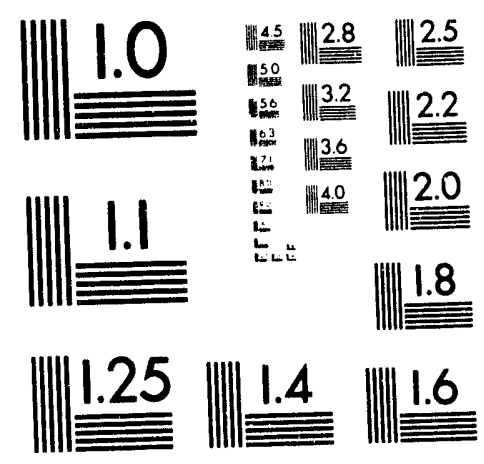



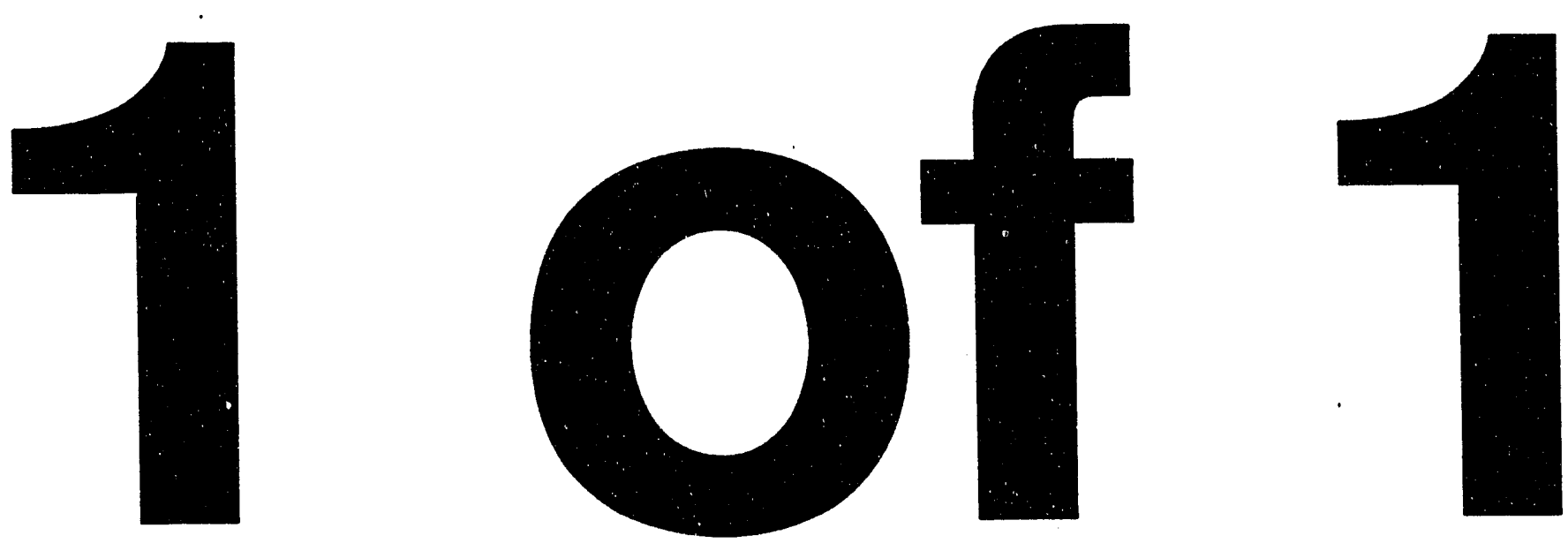


\title{
Recent Progress on MHD-Induced LosS of D-D Fusion Products in TFTR
}

\author{
S. J. Zweben, D.S. Darrow, R.V. Budny, Z. Chang*, C.Z. Cheng, \\ E.D. Fredrickson, H. Herrmann, H.E. Mynick, J. Schivell \\ Princeton Plasr.1a Physics Laboratory, P.O. Box 451, Princeton, N.J., 08543 \\ * University of Wisconsin, Madison, Wisconsin, USA
}

\begin{abstract}
Paper presented at the IAEA Technical Committee Meeting on Alpha Particles in Fusion Research

Trieste, Italy, May 10-14, 1993
\end{abstract}

\begin{abstract}
This paper reviews the recent progress made toward understanding the MHD-induced loss of D-D fusion products which has been seen on TFTR since 1988. These measurements have been made using the "lost alpha" diagnostic, which is described briefly. The largest MHD-induced loss occurs with coherent $3 / 2$ or $2 / 1$ MHD activity (kink/tearing modes), which can cause up to $\approx 3-5$ times the first-orbit loss at $I=1.6-1.8 \mathrm{MA}$, or roughly a $\approx 20-30 \%$ global loss of $D-D$ fusion products. Modeling of these MHD-induced losses has progressed to the point where the basic loss mechanism can be accounted for qualitatively, but the experimental results can not yet be understood quantitatively. Several alpha loss codes are being developed to improve the quantitative comparison between experiment and theory.
\end{abstract}

\section{MASTER}




\section{Introduction}

This paper reviews the recent progress in measurements and modeling of the anomalous MHD-induced loss of D-D fusion products seen on TFTR. A major aim of these studies is to identify the "single-particle" MHD-induced loss mechanisms for D-D fusion products in order to help isolate any new "collective" alpha loss mechanism which may occur during the TFTR D-T run. The D-D data described here comes from the 1992 TFTR run, and the D-T run is expected to start in the Fall of 1993.

Some of these results have already been summarized at the IAEA Meeting in Wurzburg, Germany in 1992[1], and in a paper on "delayed loss"[2]. The present paper will discuss these and related results more informally, with an emphasis on areas which need further work and thought. We also describe the status of the lost alpha diagnostic, and note the progress in our measurements and understanding since the first IAEA Alpha Particle Workshop at Gothenburg in $1986[3]$.

\section{The Lost Alpha Diagnostic}

These measurements were all made with the "lost alpha" diagnostic on TFTR, which was designed to measure alpha loss in D-T plasmas. The design for these detectors was first shown at the IAEA Alpha Workshop in 1986[3], and since then new prototypes or improved designs have been tested every year in situ in TFTR. At present there are four working detectors in a poloidal array at angles of $90^{\circ}, 60^{\circ}, 45^{\circ}$, and $20^{\circ}$ below the outer midplane. The first three of these are now "fixed" in position with their apertures $\approx 1 \mathrm{~cm}$ below the "RF limiter" radius, while the $20^{\circ}$ or "midplane" detector is radially movable inside the chamber. A photograph of the interior of these detectors, i.e. the aperture and scintillator arrangement, is shown in Fig. 1, and a photograph of all four of the detectors installed in TFTR is shown in Fig. 2.

All of these detectors collect the scintillation light from the fast ions hitting a thin layer of inorganic crystal powder (a technique which was first used by Rutherford in the discovery of alpha particles). Although these scintillators have a rather poor energy resolution compared to the more modern silicon detectors (such as now used by G. Martin at Tore-Supra), they have the great advantage of a high radiation damage resistance and relatively low sensitivity to neutrons, which is essential for operation with D-T plasmas. So far as we know, there is no silicon or other solid state pulse-height detector which can survive the neutron fluence of $\approx 10^{15}$ neutrons $/ \mathrm{cm}^{2}$ expected 
during the TFTR D-T run. Another great advantage of the simple scintillator is its high reliability, which has allowed it to operate untouched for 1-2 years at a time inside the TFTR vacuum vessel. In addition, its optical signal coupling is relatively immune to the high electrical and nuclear backgrounds of the tokamak.

One major diagnostic issue is the choice of scintillator (phosphor) material. These scintillators needs to have a high melting point $\left(\geq 500^{\circ} \mathrm{C}\right.$, which might be reached after a series of high power pulses), and need to be thin to minimize the radiation backgrounds (ideally $\approx 5 \mu \mathrm{m}$, just enough to stop an alpha particle at a $20^{\circ}$ angle of incidence). Originally we used the P11 phosphor, i.e. the blue $\mathrm{ZnS}(\mathrm{Ag})$ used in fast oscilloscopes, which is among the brightest of inorganic scintillators. However, this scintillator begins to "quench" at a relatively luw temperature of $\approx 150^{\circ} \mathrm{C}$, i.e. have reduced brightness for a given level of excitation, as measured with a ${ }^{244} \mathrm{Cm}$ alpha source in the laboratory[4]. Up to and including the 1990 run the detector's temperatures were below $100^{\circ} \mathrm{C}$ (as monitored by a thermocouple nearby), and scintillator overheating was not a problem. However, for 1991 the detectors were moved closer to the plasma to avoid a new limiter[5], so their temperature was expected to increase. Therefore for the 1992 run we used a P31 scintillator instead, similar to green $\mathrm{ZnS}(\mathrm{Cu})$ screen used in most oscilloscopes, which is not quite as bright but which quenches at a much higher temperature of $\approx 300^{\circ} \mathrm{C}$.

Measurements of the scintillator light output and damage vs. $\mathrm{MeV}$ ion flux have recently been made by Tuszewski using $3 \mathrm{MeV}$ protons and $3.5 \mathrm{MeV}$ alphas at the Van de Graaff at Los Alamos[6]. He found that P31 begins to saturate at flux levels below those expected for D-T alphas in TFTR (assuming that the apertures are the same size as in D-D), but that another well known green scintillator P46 (YtAl[Ce]) is linear up to the highest flux levels expected in D-T[7]. Thus P46 scintillators have been installed for the D-T run, using the same aperture sizes as used for the D-D runs. The quenching temperature of P46 is intermediate between P31 and P11, so the scintillator temperature may need to be monitored (e.g. through a UV calibration system).

The light signals from these scintillators are carried across a vacuum window to a shielded area using quartz lenses and coherent fiber bundles, and monitored there by an intensified video camera in parallel with a set of photomultiplier (PM) tubes. The camera is essential for recording the 2-D images of the scintillators (all 4 of which are arranged in the same field of view), in order to interpret these images in terms of the escaping ion's pitch angle vs. gyror dius distribution[8]. The camera sensitivity can be controlled by the voltage of the microchannel plate or by the gating time per field ( $1 \mu \mathrm{s}$ to $15 \mathrm{msec}$ ), with 42 digitized fields available per shot. There is $\approx 0.4 \mathrm{MB}$ of 
compressed video data generated by each shot, so it is also helpful to visually review the data on the VCR "backup" tape before detailed analysis.

There are presently 24 PM tubes and digitizers available for looking at selected pieces of these images at digitizer rates up to $500 \mathrm{kHz}$. During the 1992 run a set of $4 \mathrm{PM}$ tubes was arranged to look at the time dependence of the total light from each detector, which proved very valuable for studying MHD-induced loss. The remaining PM tubes are designed to look at selected pitch angle regions of various detectors during the D-T run, in parallel with the camera and integrating PM's. During the TFTR runs from 1988-1990 some of these extra PM channels were proximity-coupled to the main quartz bundle, which produced good data on fast fluctuations but sacrificed the 2-D video camera data.

Energy resolution is limited in these detectors, since it is set by the geometrical resolution of the aperture pair (as in an unfocused magnetic spectrometer) and not by the scintillator itself. Furthermore, only the ion gyroradius distribution can be determined and not the ion species, which has caused some ambiguity in ICRH minority heating experiments with multiple fast ion species[9]. Presently the measured gyroradius distribution is fit by model curves generated by a detector simulation code, which can determine relative changes in the ion energy of about $\pm 1 \mathrm{MeV}$ for $3 \mathrm{MeV}$ protons. This is good enough to distinguish a delayed loss at about half the birth energy[1,2], but not good enough to determine its detailed energy spectrum. Improved geometrical resolution could be obtained by reducing the aperture heights (presently $1 \mathrm{~mm}$ ), but only at the expense of signal level.

The absolute calibration of these detectors has been uncertain by about a factor of two or three[4], which is not very good compared to the uncertainty of about $\pm 10-20 \%$ of global neutron source measurements. This is due to a combination of several factors, including uncertainties in the light output per $\mathrm{MeV}$ ion, in the angular distribution of this light output, and in the optical calibration of the detectors. We hope to improve this uncertainty to less than a factor of two for the D-T run by measuring the light output of the P46 scintillators with actual $3.5 \mathrm{MeV}$ alphas at the Los Alamos Van de Graaff, and directly comparing this light with a reference light which was placed at the scintillator position inside the TFTR vessel. There are additional uncertainties in the interpretation of the measured escaping alpha flux due to the uncertainties in the alpha birth profile, the plasma current profile (which determines the orbit geometry), and the mechanical construction of the small apertures. 


\section{Recent Measurements of MHD-Induced Loss in TFTR}

The first signs of MHD-induced loss of D-D fusion products in TFTR came in 1988 when the array of 2-D imaging scintillator detectors was installed[10]. Increases in the fusion product loss by a factor of $\approx 3$ were seen in the $90^{\circ}$ detector at times of large MHD activity. This increased loss fluctuated in time with the frequency of the $\mathrm{MHD}$ activity in the range $\approx 2 \mathrm{~Hz}$ (nearly locked modes) to $\approx 5 \mathrm{kHz}$ (Mimov oscillations in rotating plasmas). Rapid spikes of loss at the times of sawtooth crashes were also seen in the $90^{\circ}$ detector.

Every TFTR run since then has had many discharges with very similar symptoms of MHDinduced loss, particularly at high NBI power and at high current (I $\geq 1.4 \mathrm{MA}$ ). The results can be summarized as follows[1]: (a) the D-D fusion product loss in the $90^{\circ}$ detector (per neutron) at the normal operating current range of $I \approx 1.6-2.0 \mathrm{MA}$ can increase during $\mathrm{MHD}$ by up to a factor of $\approx 3$ 5 above the MHD-quiescent level, (b) these increased loss rates seem to persist as long as the MHD activity lasts, which is often longer than the $3 \mathrm{MeV}$ proton slowing-down time of $\approx 0.2 \mathrm{sec}$. (c) this extra loss due to MHD activity can increase the total loss up to the level of first-orbit loss at $\mathrm{I}=0.8 \mathrm{MA}$, i.e. up to a calculated level of $\approx 20-30 \%$ globally (assuming its poloidal loss distribution is the same as first-orbit loss), (d) the MHD-induced loss is often observed to be strongly modulated with the MHD up to a frequency of $\approx 5 \mathrm{kHz}$, with instantaneous peak loss rates up to about 10 times the "baseline" level without MHD, and (e) qualitatively similar MHD-induced loss has been seen in all 4 detectors.

\section{a) Recent examples of MHD-induced loss}

Two examples of MHD-induced D-D fusion product loss as measured during the 1992 run are shown in Figs. 3, taken from an experiment designed to examine the effect of the plasma current profile on MHD activity and fusion reactivity in supershots[11]. For both these discharges the plasma current was $I \approx 1.6 \mathrm{MA}$, the plasma major radius was $R=2.45 \mathrm{~m}$, and the $\mathrm{NBI}$ power was $24 \mathrm{MW}$ from 3-4 sec. The fusion product loss signals shown in Fig. 3 came from PM tubes which collected the total scintillator light from each detector (the neutron/gamma backgrounds were negligible in these cases). The $20^{\circ}$ detector escaping alpha was inserted with its aperture $3.5 \mathrm{~cm}$ inside the RF limiter radius (but still well outside the plasma edge) for both these shots.

One of the discharges in Fig. 3 had "fishbone-type" $m=1, n=1$ MHD activity from $\approx 3.45$ $4.0 \mathrm{sec}$ (\#66896), but with steady plasma confinement and neutron rate from $=3.5-4.0 \mathrm{sec}$. This type showed very systematic, but small $(\approx 20 \%)$, increases in the D-D fusion product loss at the 
peak of each fishbone burst. These increases occurred simultaneously at both the $90^{2}$ and $20^{\circ}$ detectors, and lasted as long as the fishbone-type MHD $(\geq 0.5 \mathrm{sec})$. This type of shot showed the lowest level of fusion product loss at this current and NBI power.

The other discharge in Fig. 3 had gradually increasing $m=3, n=2$ mode activity from about $\approx 3.25-4.0 \mathrm{sec}$ (\#66869), with a coincident degradation in plasma confinement and neturon rat 3 from $\approx 3.5-4.0 \mathrm{sec}$. This "3/2-type" discharge showed a much larger increase of the loss at both the $90^{\circ}$ and $20^{\circ}$ detectors, e.g. the total loss (per neutron) is about 2-3 times higher for the $3 / 2$-rype than for the fishbone-type at $\approx 3.8 \mathrm{sec}$. This type of shot showed the largest level of fusion firoduct loss at this current and NBI power (discharges with $4 / 3$ modes were interinediate).

The dependencies of these same loss signals on a shorter $\approx 100 \mathrm{msec}$ time scale are shown in Figs. 4, along with the MHD activity as monitored by a Mirnov loop signal ( $\mathrm{dB}_{\mathrm{p}}$ 'dt at the wall). The fishbone-induced fusion product loss follows closely in time the envelope of the Mimov signal at both the $90^{\circ}$ and $45^{\circ}$ detectors, with a delay of $<1 \mathrm{msec}$ even at the first fishbone. This suggests that this fishbone-induced loss process is very rapid compared to the thermal energy confinement time of $\approx 150 \mathrm{msec}$. For the $3 / 2$-type discharge the iuss is only slightly modulated on a $\approx 10 \mathrm{msec}$ time scale, but apparently not in phase for the two detectors.

The dependencies of these same signals on an even faster $\approx 3-5 \mathrm{msec}$ time scale are shown in Fig. 5. For the fishbone-type discharge there is a perceptible modulation of the loss at the $90^{\circ}$ detector at the fishbone frequency of $\approx 10 \mathrm{kHz}$, but apparently not at the $20^{\circ}$ detector. For the $3 / 2$ type discharge there is a few-percent (rms) modulation of the loss at both detectors at the $3 / 2$ frequency of $\approx 20 \mathrm{kHz}$, with an $\approx 180^{\circ}$ phase difference at this frequency between the two detectors. The amplitude of this modulation approximately follows the amplitude of the Mirnov signal over the time scale of the NBI. Note that the principle decay time of the P31 scintillator is $\approx 20 \mu \sec [6]$, which tends to reduce the fluctuation level somewhat at frequencies $\geq 20 \mathrm{kHz}$.

The pitch angle distribution of the loss for these same discharges are shown in Fig. 6, here concentrating on the $90^{\circ}$ detector signals as measured by the time-integrating video camera images. During fishbone activity at 3.6-3.7 sec the additional loss appears to be localized at a pitch angle of $\approx 60^{\circ}$ (when compared with the loss during the same discharge at 3.3-3.4 sec before the start of fishbone activity). This is near the pitch angle of the passing/trapped boundary (i.e. near the fattest banana orbit), which, according to our Lorentz orbit code, is at $58^{9}$ for D-D fusion products in this discharge (given the plasma current and its profile as calculated by the SNAP code). The pitch angle distribution of the loss during the $3 / 2$ activity is more similar to that observed without MHD 
activity, with a slight tendency for the additional loss to be weighted toward high pitch angles near $70^{\circ}$, i.e. corresponding to trapped ions fairly far from the passing/trapped boundary. This suggests that the MHD induced loss to the $90^{\circ}$ detector can simultaneously consist of both passing ions (lost across the passing/trapped boundary) and trapped ions. It is also possible that the pitch angle distribution of the lost ions could fluctuate in time with the phase of the mode, as has been observed for slower MHD modes[2].

The gyroradius distribution of the loss for these same discharges is shown in Fig. 7 (for the $90^{\circ}$ detector). During fishbone activity at $3.6-3.7 \mathrm{sec}$ the additional loss has nearly the same gyroradius (i.e. energy) distribution as the loss before fishbone activity, while for the $3 / 2$ case the additional loss is weighted toward lower gyroradii, i.e. lower energies. A lowering of the peak location from $\approx 3.6 \mathrm{~cm}$ to $\approx 3.2 \mathrm{~cm}$ corresponds roughly to a lowering of the average loss energy by $\approx 20 \%$. The broad instrumental response of this detector in the gyroradius domain (see Sec. 2) makes it difficult to unfold the escaping ion energy distribution more precisely.

These two types of MHD-induced loss can be quite reproducible and persistent in highpowered NBI discharges in TFTR, and the magnitude of the loss can correlate well with the $m / n$ type over a neutron rate range $=1.5-3 \times 10^{16}$ neutrons/sec in $I \approx 1.6 \mathrm{MA}$ discharges[12]. Qualitatively similar MHD activity has been observed over the whole range of normal TFTR operation, i.e. $\mathrm{I} \approx 1.0-2.5 \mathrm{MA}$.

\section{b) Tentative interpretations and open questions}

There are several possible mechanisms which could cause the D-D fusion product loss to be correlated with background plasma MHD activity, namely: (1) modificaticn of the normal firstorbit or TF ripple loss due to MHD-induced changes in the fusion product source profile or plasma current profile, (2) magnetic fluctuation-induced radial transport of previously-confined fusion products, (3) MHD-induced effects on some presently unidentified mechanism of fusion product loss. Note that we are assuming that the D-D fursion product density and beta are too low to excite collective instabilities (which may not be true for the $\approx 100$ times larger popu. ation of $D-T$ alphas).

Increased fusion product loss of the first type (1) could be due to an $\mathrm{MHD}$-induced increase in the local D-D fusion reaction rate at radii where fusion products are lost through these effects. For example, a $20 \%$ increase in the local source rate at $r / a \approx 0.2-0.3$ in $I=1.6 \mathrm{MA}$ discharges would cause a pre..pt increase by $=20 \%$ in the loss near the fattest banana orbit in the $90^{\circ}$ detector, which could be consistent with the characteristics of the fishbone-induced loss shown in Figs. 3-4. 
However, in order to explain the increases seen during 3/2-type activity, the local source near $r / a \approx 0.3 \pm 0.1$ would have to increase by $\times 2-3$ times above that during the fishbone-type discharge, which is highly unlikely given the giobal decrease in the global neutron rate. Analysis of the neutron emission profiles for these discharges so far indicates that during MHD activity the source rate generally decreases inside $r / a \approx 0.5$ (but might increase for $r / a \geq 0.5$ ) [13].

Another classical effect on first-orbit loss could come through slow modification of the plasma current profile, which probably did occur in these experiments due to the differing plasma current evolutions (see Fig. 1). However, this is a small effect compared to the observed MHDinduced changes (an $\approx 25 \%$ decrease is expected between $1.4 \mathrm{MA}$ and 1.6 MA discharges), and also can not explain the relatively rapid changes in fusion product loss correlated with MHD changes observed in many cases. The classical TF ripple loss could also be modified by either source profile or current profile changes, but this should affect mainly the loss to the outer midplane and not to the $90^{\circ}$ detector, in contrast to the results of Fig. 3 which show a qualitatively similar MHD-induced loss at both detectors.

The second possible mechanism for MHD-induced loss is the one which we believe to be dominant in these experiments; namely, the magnetic fluctuation-induced radial transport of previously confined fusion products. Theory and modeling for this mechanism are described in Sec. 4. The present model can explain at least qualitatively the increased los $j$ with MHD activity, but there are still many quantitative features of the data which are not yet understood.

For example, in the 3/2-type discharge shown in Fig. 3 the increase in fusion product loss above the fishbone-type level peaks at $\approx 3.45 \mathrm{sec}$ when measured edge fluctuation was $\tilde{B_{\text {pol }}} \approx 0.05$ Gauss, whereas the $3 / 2$ mode amplitude continues to increase to $\widetilde{B_{p o l}} \approx 0.15$ Gauss at $\approx 3.7-4.0 \mathrm{sec}$ (the frequency of the $3 / 2$ is approximately constant over the whole discharge, so $d \widetilde{B} / \mathrm{dt} \alpha \widetilde{\mathrm{B}}$ at the wall). Thus either the $3 / 2$-induced radial transport increases less than linearly with $\widetilde{B}$, or else the perturbation depletes the accessible part of the fusion product population at a relatively low level of $\widetilde{B}$. Interestingly, the relatively small modulation level of the loss signals at the $3 / 2$ frequency follows the $3 / 2$ mode amplitude fairly closely; however, there is not yet any quantitative model for the MHD-induced modulation of the loss as a function of poloidal angle and frequency of the mode.

With regard to the pitch angle distributions of the loss to the $90^{\circ}$ detector (Fig. 6), the loss during fishbone-type MHD near the passing/trapped boundary could be explained by the $(1,1)$ MHD activity, which could push some counter-passing D-D fusion products across the passing- 
trapped boundary (which happens to be near the $\mathrm{q}=1$ surface in this plasma). This would cause these ions to be trapped and immediately lost, which is consistent with the very small time delay observed between the MHD activity and the loss. Only a small fraction $(\approx 10 \%)$ of the counterpassing ions would need to be lost to explain the $\approx 20 \%$ increase in the loss. The loss at higher pitch angles during 3/2-type activity (and also some of the fishbone-type shots) implies that the MHD can also deconfine trapped ions, which is qualitatively consistent with the magnetic perturbation model of Sec. 4. However, a quantitative understanding of these pitch angle distributions ys. poloidal angle and MHD mode type has not yet been obtained.

The loss of low-energy components during 3/2-type activity shown in Fig. 7 and elsewhere[2] shows that this type of $\mathrm{MHD}$-induced loss is not simply due to a modification of the prompt first-orbit or TF ripple loss. The magnetic perturbation model of Sec. 4 predicts that lower energy ions can be deconfined by $\mathrm{MHD}$, but only inside smaller stochastic regions. However, a quantitative explanation of the observed 3/2-type loss at low energy (and the apparent absence of low energy components in the fishbone-type case) has not yet been obtained. Note that this loss of low energy components may also imply a relatively slow loss process $(\approx 0.1 \mathrm{sec})$, which could complicate the temporal relationship between the $\mathrm{MHD}$ level and the fusion product loss.

The third possible cause of MHD-induced loss mentioned above involves the indirect effect of the MHD on some presently unidentified mechanism of fusion product loss. Such a mechanism is not entirely academic, since an anomalous non-fluctuating "delayed loss" component at about half the birth energy has already been identified in $\mathrm{I}=1.6 \mathrm{MA}, \mathrm{R}=2.45 \mathrm{~m}$ discharges (such as those described above), with or without MHD activity[1,2]. For MHD-quiescent (e.g. low NBI-power) discharges this delayed loss begins $\approx 0.2 \mathrm{sec}$ after the start of NBI, and is constant in time from $\approx 0.4-1.0 \mathrm{sec}$ after the start of NBI. It is interesting that the delayed loss to the $90^{\circ}$ detector can either increase dramatically or disappear almost entirely during times of strong MHD activity[2], as if the MHD activity were modifying that loss channel. Therefore it is also possible that the MHDinduced loss for the 3/2-type mode could be due to an increase in this delayed loss process.

\section{c) Other Types of MHD-Induce L Loss}

MHD-induced loss was also observed during the 1992 TFTR run during sawtooth crashes and just before major disruptions[1]. The loss at sawtooth crashes is usually a fast spike $(\approx 10-100$ $\mu \mathrm{sec}$ wide) localized in pitch angle near the passing/trapped boundary, which can occur at every sawtooth crash during NBI (usually there is no more than one sawtooth crash during NBI in supershots, but several during NBI in L-mode plasmas). A systematic study of these spikes has 
not yet been made, since they seem to cause a negligible total loss (although they might well redistribute charged fusion products within the plasma). The pre-disruptive MHD-induced loss rate, which can occur during locked modes about as much as $\approx 50 \mathrm{msec}$ before the plasma current quench, can be up to $\approx 50$ times the level of the MHD-quiescent loss rate. This process has been seen to expel up to $\approx 10 \%$ of the existing $D$ - $D$ fusion products over the $\approx 10$ msec preceding a current quench[1]; thus this phenomenon could potentially cause a special wall-loading problem for alpha-heated reactors. Note that measurements and modeling of the loss of NBI ions and ICRH tail ions due to collective fast ion MHD instabilities in TFTR D-D is described in a companion paper by Darrow at this meeting[14].

\section{Modeling of MHD-Induced Loss at PPPL}

It is important to develop an understanding of the mechanisms of this MHD-induced loss in order to correctly infer the consequences for alpha heated tokamaks such as ITER, which will have an $\approx 3$ times larger ratio of the plasma size to the alpha gyroradius, and so will have better (classical) confinement of alphas than does TFTR. Recent modeling has aimed to understand the interactions between charged fusion products and helical magnetic islands, which are thought to be a basic component of what we normally call "MHD activity" in tokamaks, and to apply numerical models of this physics to explain the TFTR fusion product loss data such as described above.

The mechanism of this interaction occurs through perturbed ion drifts across the toroidal field due to the "magnetic flutter" effect (proportional to the local $\tilde{B}_{\Gamma} / B_{T}$ ), and also to grad-B drifts in the perturhed fields (proportional to sqrt $\left(\tilde{B}_{r} / B_{T}\right)$ ). It has been found that there is a threshold level of the perturbed magnetic field magnetic field above which either passing or trapped ion orbits can become stochastic[15]. Since this threshold depends on the mixing of the $m=1, n=0$ orbit shift with the $\mathrm{m} / \mathrm{n}$ structure of the magnetic perturbation, this threshold is generally lower for higher energy ions, which stray farther from the magnetic field lines. Thus fusion product ions are more likely to become stochastic than lower energy neutral beam ions, given the same perturbed magnetic field structure.

This stochastic fast ion motion can cause a very rapid radial transport of fusion products, which could lead to rapid loss if this stochastic particle motion region reaches the wall. However, this MHD-induced stochastic orbit region could also be localized within the plasma where the magnetic perturbation is largest, whereas the analogous stochastic TF ripple region[16] always causes fast ion loss to the wall due to the radially-increasing TF ripple strength. 
This theory has been incorporated into a guiding center code "GC3" to check the analytical theory and to apply the results to specific TFTR cases[17]. So far test cases have been run to evaluate the total MHD-induced loss of $3.5 \mathrm{MeV}$ alphas for an $\mathrm{I}=2 \mathrm{MA}$ and $\mathrm{R}=2.45 \mathrm{~m}$ TFTR discharge vs. the amplitude of various assumed $(m, n)$ modes, as shown in Fig. 8[adapted from Ref. 1]. Note that only the magnetic flutter term (and not the grad-B drift term) was used for this run, and that collisions, TF ripple, and the Shafranov-shift were also not included. Each point in Fig. 8 represents a 5 hour run on a Sun workstation in which 300 ions were followed for 3000 poloidal transits ( $\approx 10 \mathrm{msec}$ real time for alphas).

During these runs most of the MHD-induced lost alphas (above the first-orbit level of $\approx 4$ $6 \%$ ) hit either near the outer or inner midplane where the excursions of the orbit from the magnetic flux surface were largest (for co- or counter-passing ions, respectively), although some of the counter-passing ions did cross the passing/trapped boundary to hit the bottom[15,17]. Most trapped ions which were lost through stochastic diffusion tended to escape near the outer midplane where their orbits first intersect the wall, as in stochastic TF ripple diffusion[16]. For the cases cf single $(\mathrm{m}, n)$ modes the total loss becomes comparable to the first-orbit loss at $\tilde{B}_{\Gamma} / B_{T}=1-2 \times 10^{-3}$ (as evaluated near the rational surface), roughly independent of mode number.

The estimated internal $\tilde{B}_{r} / B_{T}$ for the two discharges of Sec. 3 are also shown in Fig. 8 (as estimated for the region near the rational surfaces from edge magnetic fluctuation levels). This model apparently does not agree well with the data for the 3/2-type MHD of Sec. 3, since the model predicts a negligible $3 / 2$-induced increase above the first-orbit loss level at the estimated $\tilde{B}_{\Gamma} / B_{T} \approx 10^{-4}$ for this case, and instead predicts that a level of $\approx \tilde{B}_{\Gamma} / B_{T} \approx 3 \times 10^{-3}$ would be needed to cause the observed factor of $\approx 2-3$ increase above the first-orbit loss. However, the model appears to agree better with the data for the fishbone-type MHD of Sec. 3, since the model predicts an $\approx 50 \%$ increase above the first-orbit loss level for a $(1,1)$ mode at the estimated $\tilde{B}_{\Gamma} / B_{T} \approx 10^{-3}$ for this case, which is near the obsen $i z 20 \%$ increase.

There are several inadequacies in the present level of comparison between the observed and calculated loss. First, the estimated internal magnetic fluctuation levels are quite uncerain (perhaps by a factor of 2), since they are derived from extrapolated edge measurements. Second, the comparison should actually be done for the local loss (e.g. at the $90^{\circ}$ detector), rather than for the total loss; however, this was not possible given the poor statistics for these guiding center runs. Furthermore, it is possible that small additional $(m, n)$ components could dramatically increase the calculated loss due to a reduction in the stochastic threshold. Other complicating factors include the 
uncertainties in the plasma current profile and the fusion product source profile (including possible fluctuations in the local source rate due to the MHD itself), and the possible "synergistic" effect of TF ripple and collisions, which were not included in the calculations of Fig. 8, e.g. that MHDinduced loss brings ions out to a radius where the TF ripple causes them to be lost.

The relatively poor statistics of these guiding center code calculations has led to the development of simpler codes which can assess the basic effects more quickly. One of these is a generalization of MAPLOS, which was originally written to calculate the poloidal distribution of fusion product loss due to TF ripple in TFTR[18]. This code keeps track of the ion's radial position only once each poloidal transit, and so can foilow $\mathbf{2 0 0 , 0 0 0}$ orbits in a few hours of CRAY time. This permits a more accurate assessment of the poloidal and pitch angle distribution of the lost ions, which are difficult to deduce accirately with only $\approx 300$ ions (only $\approx 20-30 \%$ of which may be lost). A sample calculation from this code is shown in Fig. 9, in which the perturbation step sizes per poloidal transit were chosen to approximate those in an MHD-active discharge, e.g. the maximum step size was $\approx 4 \mathrm{~cm}$ per bounce for trapped ions, and $\approx 2 \mathrm{~cm}$ per transit fc $s$ passing ions. The effect of an ad hoc variation in the radial localization of the MHD on the poloidal distribution of the loss can be clearly seen. Obviously, the fidelity of this type of code to the experimental situation depends on the accuracy of the choice of step sizes per transit, which will be improved by input from both experiment (e.g. MHD amplitudes) and theory (e.g. calculated step sizes).

Another way to quickly assess the effects of MHD on high energy ion loss is to evaluate the analytical stochastic threshold itself for particular cases by specifying the particle energy, MHD mode amplitudes, and the plasma current profile. This method is being incorporated into a new code STOCHI.OS, which can evaluate the total loss up to $\approx 1000$ times faster than the guiding center code. Preliminary comparisons between STOCHLOS results and GC3 are shown in Fig. 10 for two types of MHD perturbations, where the alpha source in both cases was assumed to be localized at $r / a=0.4$. Good agreement is found between the two codes with respect to the total loss vs. $\tilde{B}_{\Gamma} / B_{T}$. For a distributed source, the code can also identify the regions of $\mu \mathrm{B} / \mathrm{E}$ (normalized magnetic moment) and $r / R_{0}$ from which the loss occurs, as shown at the bottom of the figure. 


\section{Retrospective and Outlook}

\section{a) Status of the lost_alpha diagnostic}

The lost alpha diagnostic on TFTR has :vrrked well since the present poloidal array of 2-D imaging scintillator detectors was installed in 1988. The flux of escaping D-D fusion products has been measured for nearly every TFTR shot with NBI or ICRH heating (>10,000 shots), and the quality of much of this data has been excellent (signal/noise $>10$ ). The diagnostic has evolved more or less as envisioned at the 1986 IAEA Alpha Workshop, with major improvements coming through the use of a movable midplane probe[19], and removal of the $3 \mu$ foil on this probe to allow measurements of $100 \mathrm{keV} \mathrm{NBI}$ ions[20].

The greatest insufficiency in this system appears to be the relatively small number of detectors (i.e. 4), which limits the accuracy with which the global loss of fusion products can be evaluated. For example, there may undetected loss near the inner midplane, or slow anomalous losses with very small racial step sizes which may not reach the present fixed apertures $\approx 1 \mathrm{~cm}$ below the shadow of the RF limiters (although in some cases these detectors can be magnetically in front of the RF limiters due to the TF ripple-induced motion of the field line). Also, the loss is presently measured only over a range of pitch angles $\approx 45^{\circ}$ to $85^{\circ}$ with respect to the co-going toroidal field (centered around the passing/trapped boundary), so we can see neither the TF rippletrapped ions (very near $90^{\circ}$ ) nor the loss of nearly parallel ions (e.g. due to TAE modes). A set of $\approx 50$ detectors of the same design could probably insure complete coverage of lost alphas. For future applications it may be valuable to supplement a set of fixed scintillator detectors with scintillators on movable probes[19,20] or time-integrating alpha collectors[21].

The outlook for these detectors for the TFTR D-T run is promising, but not certain. The expected saturation of the bright $\mathrm{P} 31(\mathrm{ZnS})$ scintillators used for the 1992 run during the $\approx 100$ times larger alpha fluxes expected for the D-T run[6] has forced us to either reduce the aperture size or change the scintillator. Various scintillators were investigated, and the optimum for this application appears to be P46. However, the main difficulty with P46 is its very low efficiency, roughly 30 times lower than $\mathrm{P} 31$ for alpha excitation; thus the system as a whole becomes more susceptible to radiation-induced backgrounds such as those due to flourescence in the quartz fiber bundles[22]. Hopefully, the resulting signal-to-background level will be $\geq 1$ in $D-T$, since the fiber-optic flourescence in D-D plasmas was $<<10 \%$ of the D-D signal levels. 
These scintillator detectors might also work in ITER, since the P46 scintillator should be rugged enough to survive for a while at that high neutron flux; however, the thermal heat load and optical signal transmission problems need to be solved. More rugged thermal detectors or removable He analysis samples might be better for in-vessel alpha measurements in ITER, but would not provide such detailed information on the pitch, energy, and time dependencies.

\section{b) Status of measurements of MHD_induced loss.}

The basic observations of MHD-induced loss have been quite consistent since 1988 when the present lost alpha system was first installed. In general, the largest MHD-induced loss occurs during high-power (usually high beta) limit of TFTR operation, which has been approached every run year at successively higher NBI power and higher plasma current. The addition of ICRH heating does not appear to change this behavior qualitatively, except for new Alfven-wave MHD created by the minority ion tail itself.

For the TFTR D-T run, this diagnostic will focus on a search for collective alpha instabilities in D-T plasmas. An important part of this effort will be to carefully investigate whether any MHD-induced alpha loss during $D-T$ is due to some new collective alpha effect, or simply due to the usual "single-particle" MHD-induced effects such as discussed in Sec. 3. Any new collective alpha instability which causes a substantial alpha loss may have an effect on the ITER plasma or first wall design.

It is interesting to note that the predictions of alpha particle betas and densities for the TFTR D-T experiment which were made for the 1986 Alpha Workshop paper (before the discovery of TFTR supershots) are not far from the best predictions made now, e.g. Mikkelsen predicted $\beta \alpha(0) \approx 0.4 \%$ for the medium density case in 1986, and Budny predicts $\beta_{\alpha}(0) \approx 0.3 \%$ now [23]. This is mainly because the alpha particle parameters depend upon the plasma temperature, and not directly on the energy confinement times or fusion energy gains[24]. The currently anticipated TFTR D-T supershots will have an estimated $\approx 1-2 \mathrm{MW}$ of alpha heating, which should lead to a measurable central electron heating[25].

\section{c) Status of modeling of MHD-induced loss}

Real progress has been made in understanding the mechanisms of the MHD-induced loss of fusion products since the first Alpha Workshop in 1986, although there is still much to be done to understand the existing D-D experimental results. Numerical work along these lines is being 
pursued by several groups, both for the quasi-static background-plasma-induced MHD modes described above and also for higher frequency TAE-type modes[26-29].

A distinction should be probably be drawn between alpha loss driven by alpha-induced "collective" MHD activity, which naturally tend to transport alphas in order to flatten their driving spatial gradient, and alpha loss driven by "single-particle" background plasma-induced MHD activity (such as discussed in Sec. 3), in which the fast ions do not directly affect the instability. Although the physics of the wave-particle interaction may be similar, the non-linear development and the eventual effects on alpha loss may be different. Both of these effects could occur for alpha particles in TFTR D-T or ITER. Two good reviews have been written recently on collective fast ion instability experiments[30] and theory[31].

A major issue for the future will concern the practical consequences of MHD-induced alpha loss for the design of ITER. The most likely effect of such alpha loss will be to increase the heat load in localized regions of the first wall or divertor plate -- obviously, these regions need to be carefully identified and protected. Pioneering efforts in this direction have recently been made by the JT-60U group, which has measured the 2-D pattern of heat loads on their outer carbon wall with thermocouples and infrared TV measurements, and have identified this heat loss as due to TF ripple loss of NBI ions using their orbit following Monte Carlo code[32].

The alpha loss due to MHD will probably be more variable and more difficult to calculate, given the complicated structure of MHD effects in time and space, and the potentially non-linear interactions between the alpha loss and any collective alpha instabilities. Recent work on DIII-D has shown that the loss of NBI ions during TAE activity occurs mainly near the outer midplane[33], but this may not be true for alpha particles in ITER. Simulation and calculation of the $\mathrm{MHD}$-induced alpha heat loss onto the first-wall and/or divertor seems to be an important area for further work in this field.

Acknowledgments: We thank for discussions on these topics our colleagues at PPPL: C. E. Bush, H. Biglari,, L. Chen, H. P. Furth, R. Hawryluk, L. Johnson, K. McGuire, D. Meade, D. Mikkelsen, J.D. Strachan, R.B. White, and K.M. Young, and also R. Boivin and D. J. Sigmar from MIT, and M. Tuszewski from LANL. This work was performed under DoE contract No. DE-AC02-CHO-3073. 


\section{References}

[1] S.J. Zweben, et al, LAEA Wurzburg, Paper A-6-3 (1992)

[2] S.J. Zweben, D.S. Darrow, E.D. Fredrickson, and H.E. Mynick, Princeton Plasma

Physics Laboratory Report PPPL-2864 (1993), to be published in Nuclear Fusion, 1993

[3] S.J. Zweben, Physics Scripta Vol. T16, 1987, 119

[4] R. Boivin, et al, Rev. Sci. Inst. 63(10), 1992, 4418

[5] S. J. Zweben, et al Rev. Sci. Inst. 63(10) 1992, 4565

[6] M. Tuszewski, et al, Rev. Sci. Inst. 63(10) 1992, 4542

[7] M. Tuszewski and S. Zweben, Rev. Sci. Inst., Aug. 1993

[8] S.J. Zweben, R.L. Boivin, C.-S. Chang, et al, Nucl. Fusion 31(12), 1991, 2219

[9] S.J. Zweben, G. W. Hammett, R.L. Boivin, et al, Nucl. Fusion 32(10), 1992, 1823

[10] S.J. Zweben, J.D. Strachan, R. Boivin, et al, EPS Venice, Italy, 1989, 39

[11] R.V. Budny, PPPL, private communication (1993)

[12] Z. Chang, et al, EPS Lisbon, 1993, to be published

[13] L. Johnson, PPPL, private communication (1993)

[14] D.S. Darrow, et al, this meeting

[15] H.E. Mynick, Phys. Fluids B5, 1993, 1471

[16] R.B. White and H.E. Mynick, Phys. Fluids B1, 1989, p.980

[17] H. Mynick, to appear in Phys. Fluids B, 1993

[18] R. Boivin, R.B. White, and S.J. Zweben, Nucl. Fus. 33(3), 1993, 449

[19] R. Boivin, et al, Rev. Sci. Inst. 61, 1990, 3208

[20] D. Darrow, et al, Rev. Sci. Inst. 63(10) 1992, 4562

[21] R. Bastasz, et al, Rev. Sci. Inst. 61, 1990, 3199

[22[ A. T. Ramsey and K.W. Hill, Rev. Sci. Inst. 63, 1992, 4735

[23] R. Budny, et al, Nuclear Fusion 32, 1992, 429

[24] H. Furth, et al, Nuclear Fusion 30, 1990, 799

[25] R. Budny, et al, EPS Lisbon, 1993, to be published

[26] E. Bittoni and M. Hagei, Fusion Technology 22, 1992, 461

[27] D. J. Sigmar, et al, Phys. Fluids B4, 1992, 1506

[28] C.Z. Cheng, et al, IAEA Wurzburg 1992

[29] H. Berk, B. Breizman, and H. Ye, Phys. Fluids B5, 1992, 1506

[30] W.W. Heidbrink and G. Sadler, to be published in Nuclear Fusion

[31] H. Biglari, to be published in Physics of Fluids

[32] K. Tobita, et al, Phys. Rev. Lett. 69, 1992, 3060

[33] H.H. Duong, et al, General Atomics Report GA-A21038 (1993) 


\section{Eigure Captions}

1. Photo of the scintillator and aperture configurations for the three "fixed" lost alpha detectors installed in TFTR for the 1992 run period (the configurations were very similar for 1988-1991). These detectors differ only in the ang:- between the scintillator plane and the axis of the probe shaft ,in order to match the local vessel shape (i.e. left-to right $45^{\circ}, 60^{\circ}$, or $90^{\circ}$ ). The quartz scintillator plate with the white phosphor coating is protected by a $1 \mathrm{~mm}$ thick tantalum $\mathrm{x}$-ray shield.

2. Photo of the three "fixed" lost alpha detectors installed at the bottom of the TFTR vessel, along with the movable $20^{\circ}$ probe inserted through the "midplane" port on the right. The mushroom shaped carbon heat shields covering the fixed detectors are water cooled on order to keep the scintillator temperature below $\approx 150^{\circ} \mathrm{C}$. Fusion products approach the detectors from below (in this photo) and enter the fixed probe apertures through a small cut-out in the carbon armor.

3. MHD-induced loss of $D-D$ fusion products during NBI for $R=2.45 \mathrm{~m}, I=1.6 \mathrm{MA}, 24 \mathrm{MW}$ NBI discharges in TFTR. These shots differ in their type of MHD activity, most likely due to the different plasma current evolution before NBI (and not the slightly different final current). The MHD-induced loss in the fishbone-type shot (\#66896) starts at about $3.45 \mathrm{sec}$, while the MHDinduced loss in the 3/2-type shot (\#66869) appears to start at $\approx 3.25 \mathrm{sec}$. The D-D fusion product loss at both the $90^{\circ}$ and $20^{\circ}$ (midplane) detectors increases by $\approx 20 \%$ at each fishbone, and by $\approx x 2$. 3 during 3/2-type MHD. Without the MHD activity the escaping fusion product signals follow a time dependence similar to the envelope of the fishbone-type case, but without the fishbone bursts[2].

4. Comparison over a time scale of $\approx 100 \mathrm{msec}$ of the $\mathrm{MHD}$ activity and fusion product loss for the same two shots shown in Fig. 3. The fishbone-induced D-D fusion product loss begins at the first fishbone, and shows very little delay with respect to the fishbone magnetic envelope $(<1 \mathrm{msec})$. The 3/2-type loss evolves slowly over the NBI duration, with only small modulations on $a \approx 10$ msec timescale. Note that the high frequency fishbone magnetic $\mathrm{dB} p / \mathrm{dt}$ fluctuations are not accurately represented on this timescale due to aliasing.

5. Comparison over a timescale of $\approx 3-5 \mathrm{msec}$ of the $\mathrm{MHD}$ activity and fusion product loss for the same two shots shown in Fig. 3. The escaping $D-D$ fusion product signals are only weakly modulated at the tishbone frequency of $\approx 10 \mathrm{kHz}$, or the $3 / 2$ mode frequency of $\approx 20 \mathrm{kHz}$. The inferred amplitude of the magnetic perturbation near the $q=1$ surface during the fishbone is roughly $\tilde{B_{r}} / B_{T} \approx 10^{-3}$, and the amplitude of the $3 / 2$ mode is roughly $\tilde{B}_{r} / B_{T}=10^{-4}$ near the $3 / 2$ surface. 
6. Pitch angle distributions of the loss at the $90^{\circ}$ detector for the discharges of Fig. 3. The MHDinduced loss during fishbone activity seems to be localized near the passing/trapped boundary (when compared with the same shot before the start of fishbone activity), which in this discharge is near $\approx 58^{\circ}$. The loss during 3/2-type activity appears to be spread out over a wider range of pitch angles, including those corresponding to the loss of D-D trapped fusion products near $70^{\circ}$.

7. Gyroradius distributions of the loss at the $90^{\circ}$ detector for the discharges of Fig. 3. The loss during fishbone activity has nearly the same gyroradius (i.e. energy) distribution as the loss before fishbones (which includes a non-fluctuating delayed loss component). The loss during 3/2-type activity has an additional low-energy component which makes the total loss peak at a lower gyroradius. Note that the instrumental resolution causes a considerable broadening of the gyroradius distribution for monoenergetic ion loss.

8. Calculations of total MHD-induced loss for 2 MA TFTR discharges (adapted from Ref. 1). The loss here is calculated using a guiding center code GC 3 for separate $3 / 2$ and $1 / 1$ perturbations. Each point corresponds to a Monte Carlo run of 300 alpha-like particles for 3000 poloidal transits $(\approx 10 \mathrm{msec})$. The estimated magnetic fluctuation levels for the fishbone and $3 / 2$ type fluctuations of Figs. 3-5 are indicated (as estimated near the maximum of their mode amplitudes)

9. A MAPLOS calculation, with a simple model of MHD perturbations, of the poloidal distribution of $3.5 \mathrm{MeV}$ alphas reaching the wall of TFTR. Two variations of the MHD perturbation are compared. The radial step size per poloidal transit is assumed to peak either near $r / a \approx 0.4$ or 0.9 , with an arbitrarily chosen shape (trapped ions were assumed to have twice the radial step size as passing ions). The total alpha loss fraction was $\approx 30 \%$ in this case. These results illustrate the potential sensitivity of the poloidal distribution of MHD-induced loss to the structure of the internal magnetic perturbation.

10. Preliminary comparison between TFTR alpha loss calculations done with the guiding center code GC3 and the analytic code STOCHLOS. The alphas are assumed to be born at $\mathrm{r} / \mathrm{a}=0.4$ in both cases, one assuming a single $(2,1)$ mode and the other a mixed $(2,1)+(3 / 2)$ magnetic structure. The calculated loss fractions increase sharply above the stochastic threshold of $\tilde{B}_{r} / B_{T}$ $=3 \times 10^{-3}$ for both models; however, the STOCHLOS calculation was $\approx 1000$ times faster. The distribution of lost ions for a distributed source vs. their birth radius and pitch angle is also shown. 


$$
\text { 承 }
$$



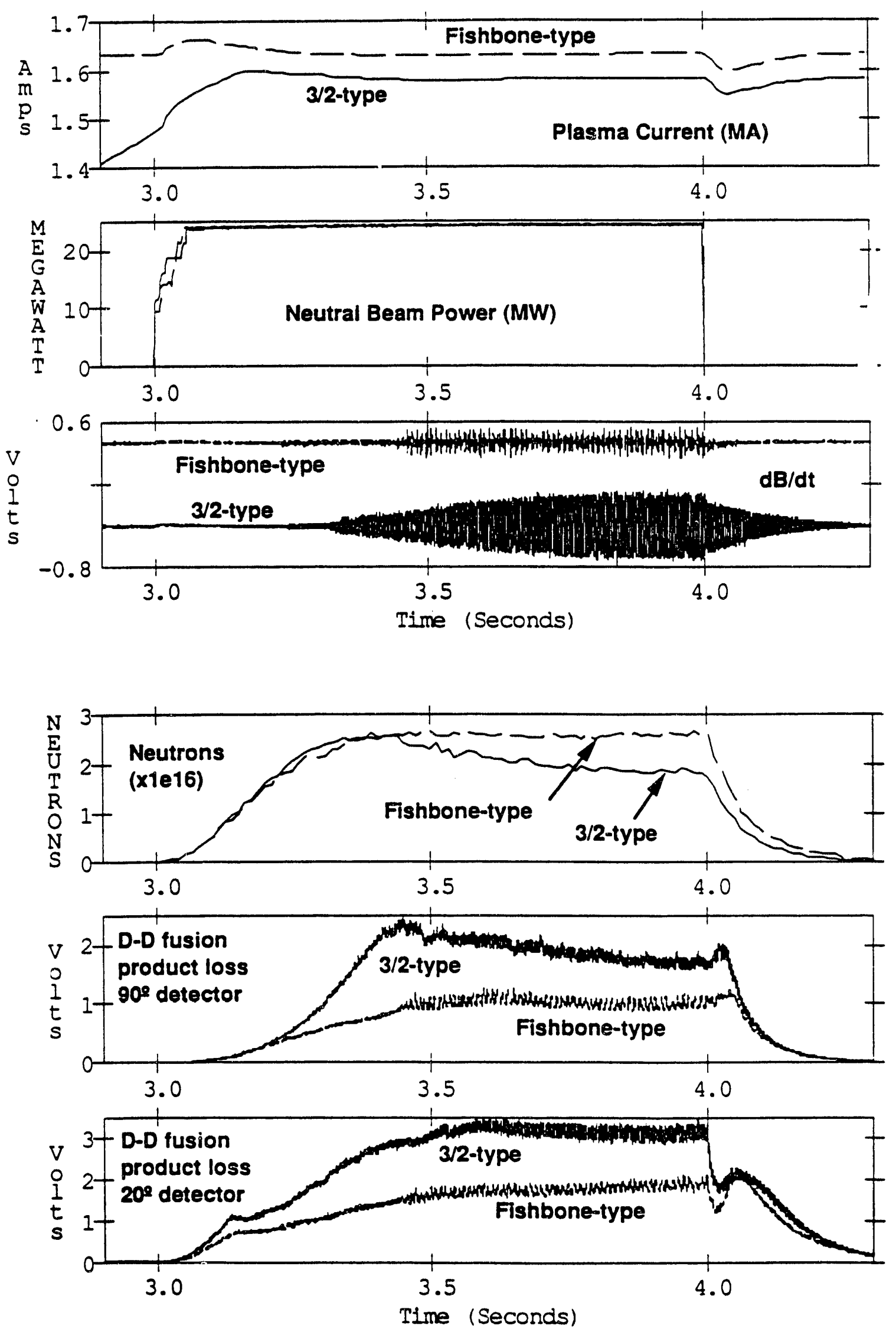

Fig. 3 



Fỉg. 4 


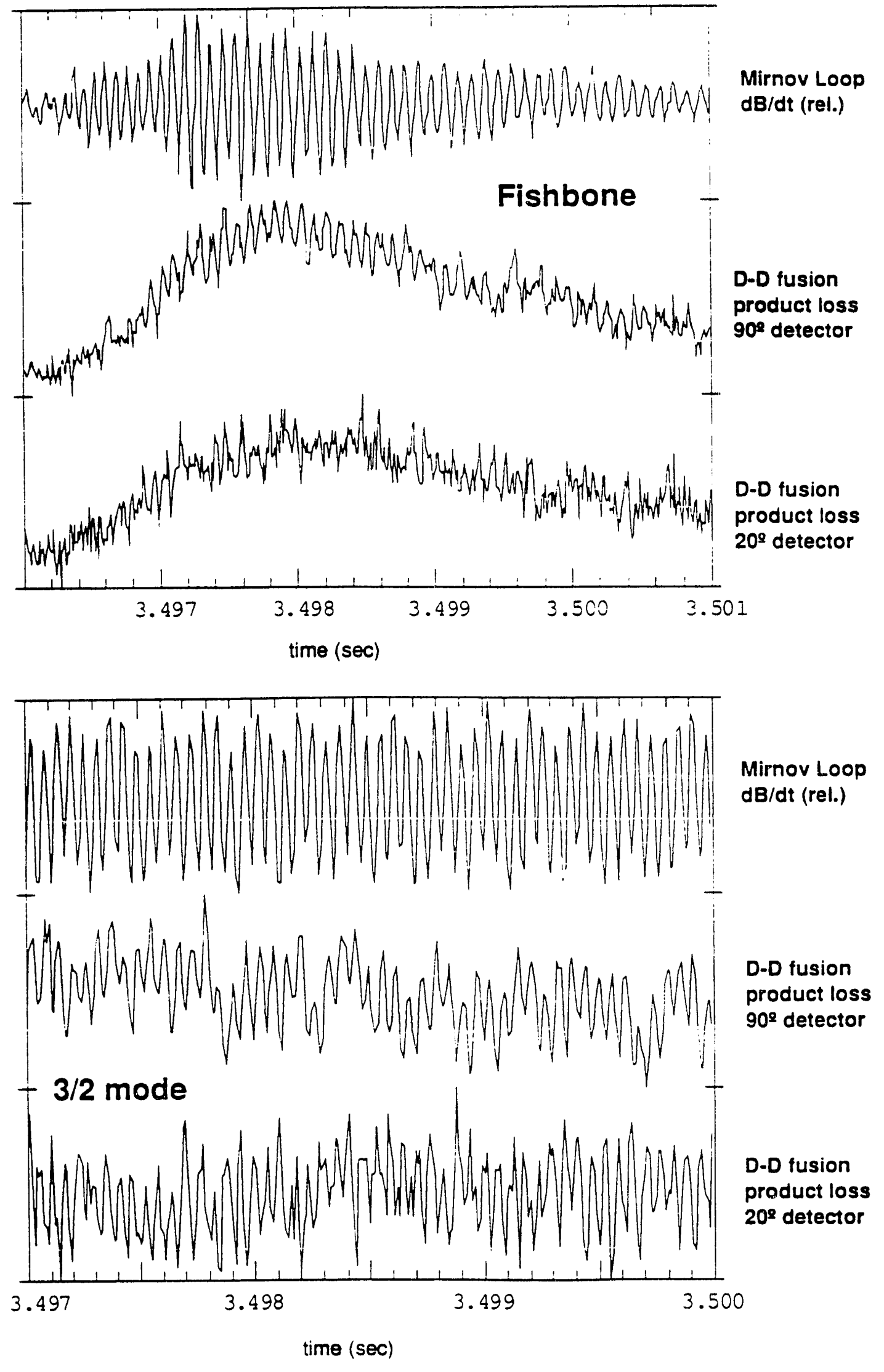

Fig. 5 


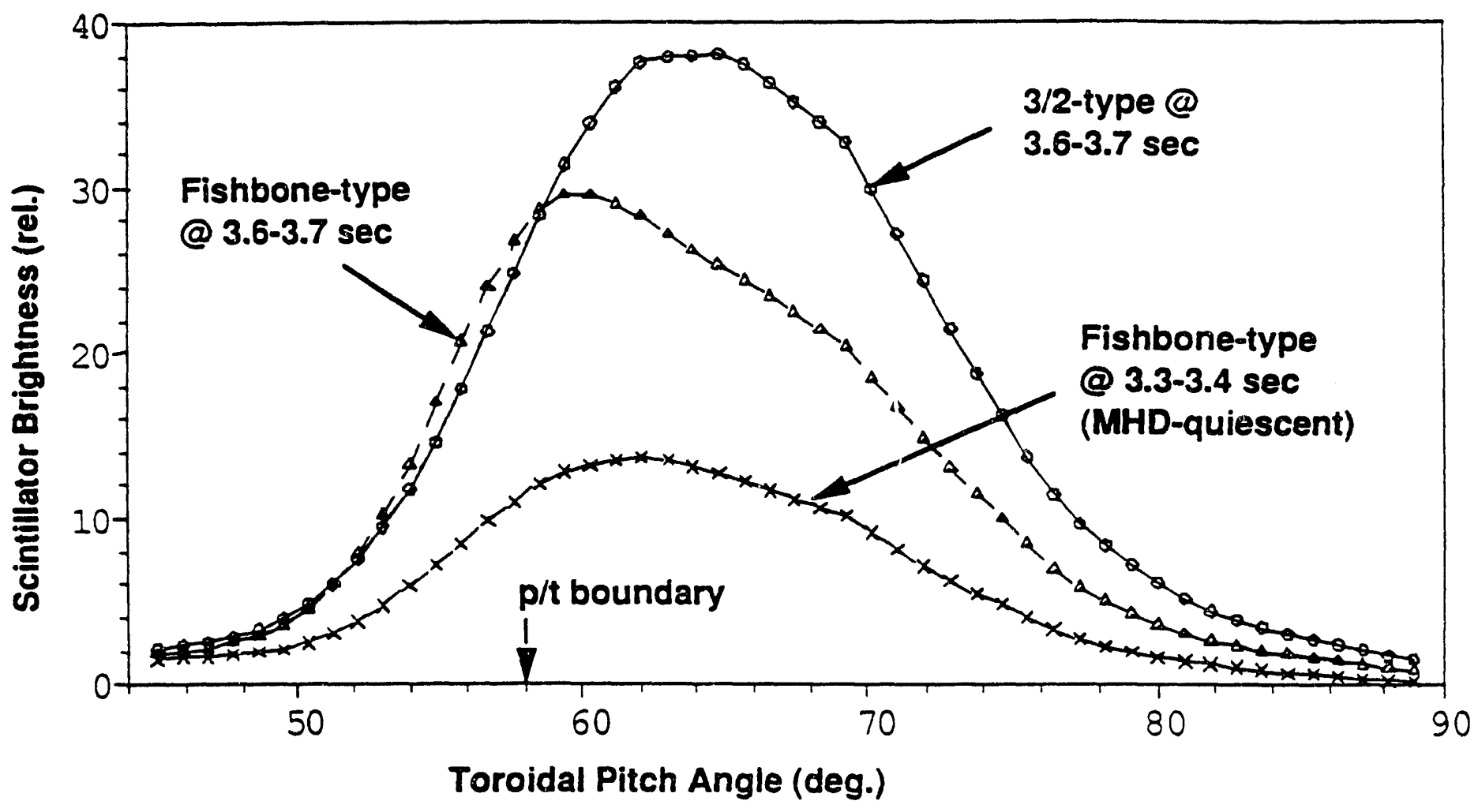

Fig. 6

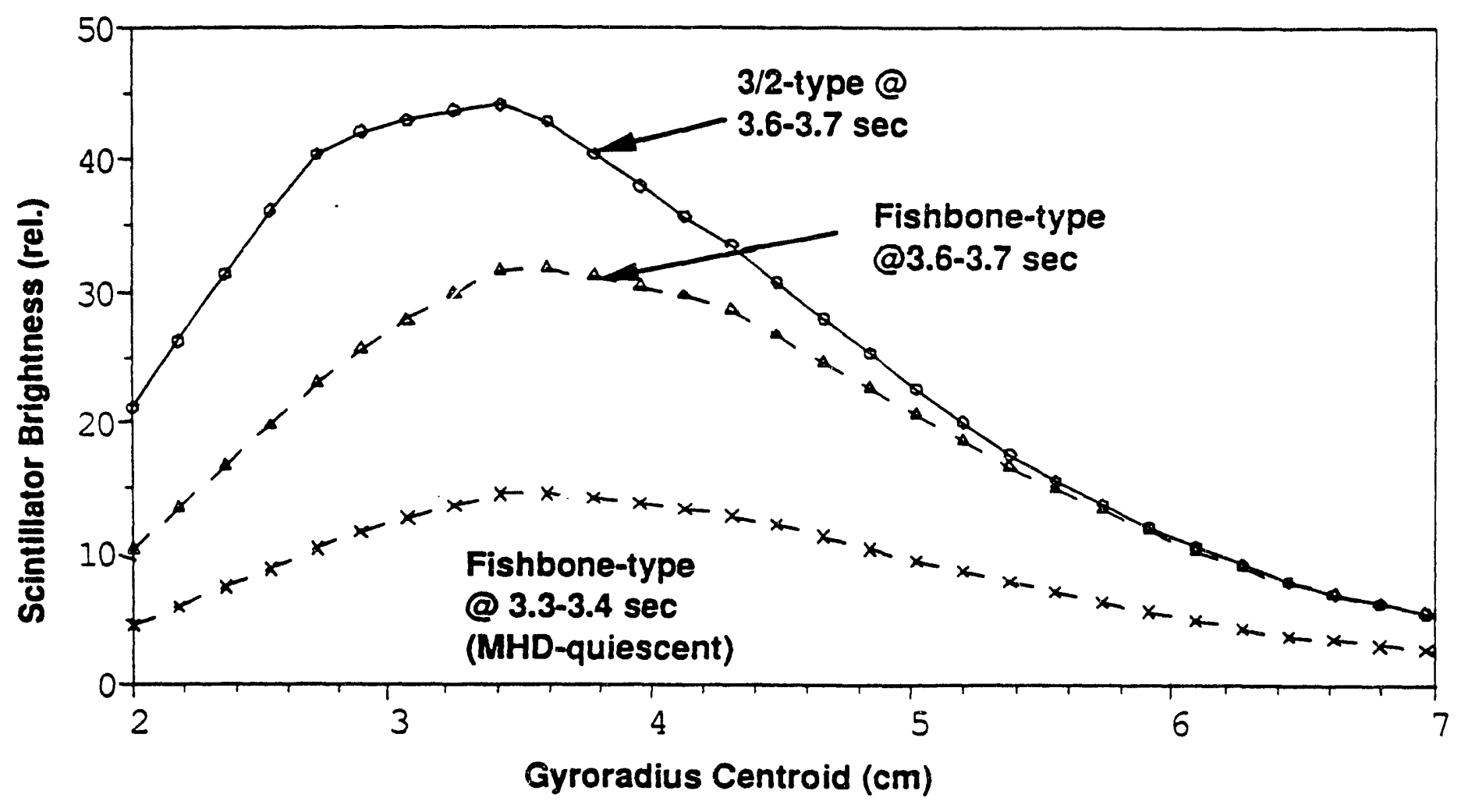

Fig. 7 



Fig. 8 

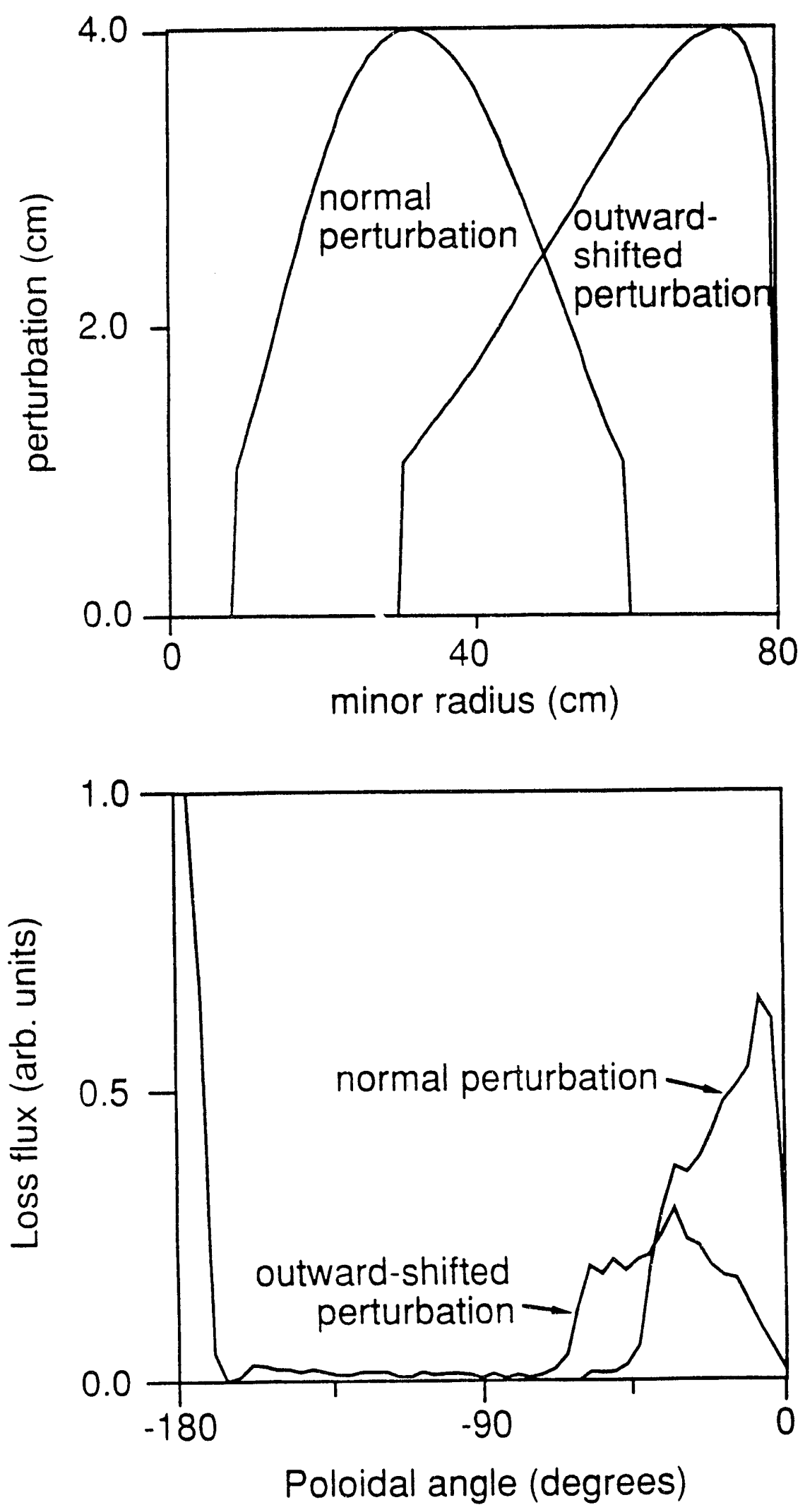

Fig. 9 

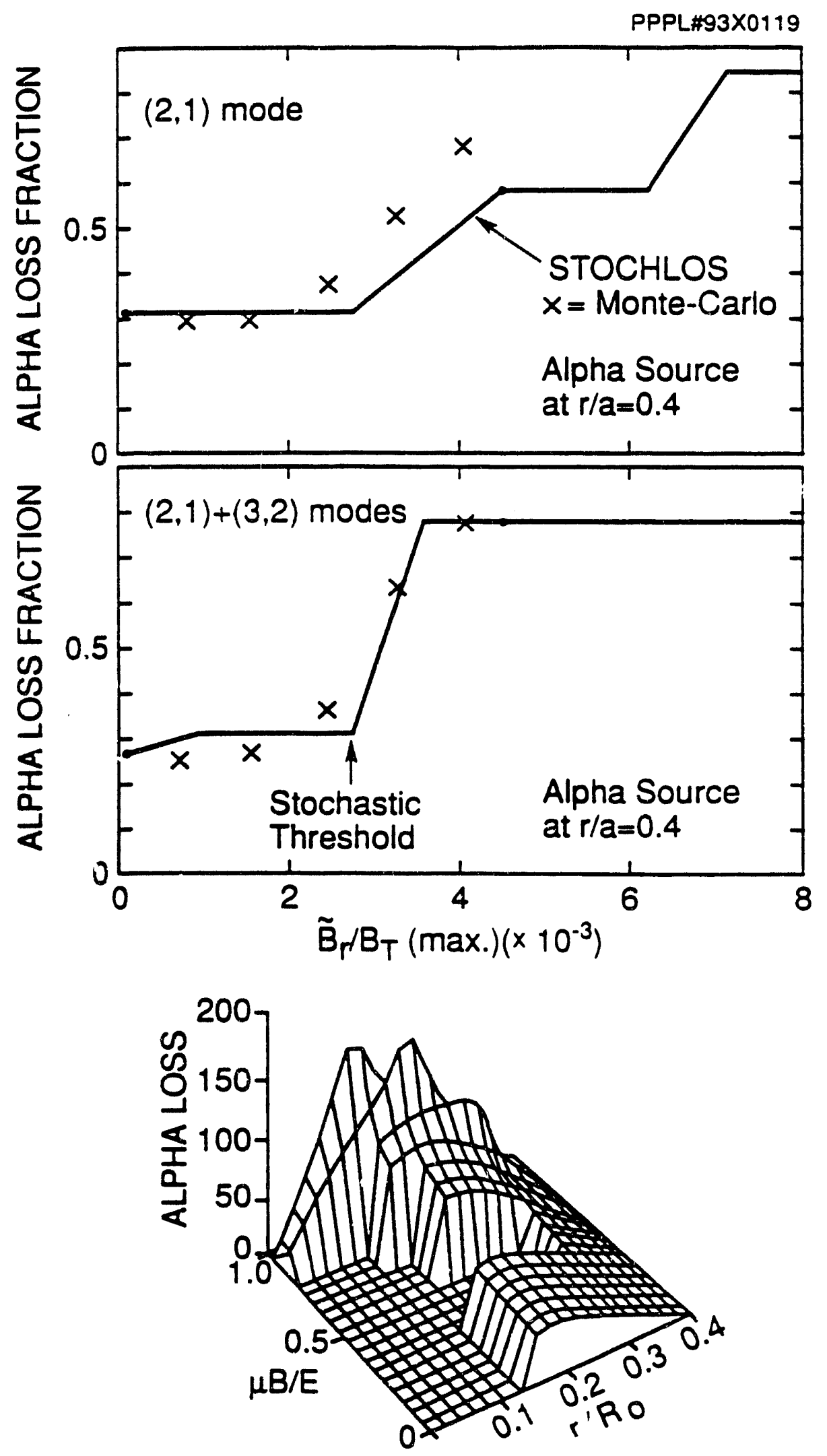

Fig. 10 
Dr. F. Peoboni. Univ. of Woltongong. AUSTRALIA Prot. M.H. Brennan, Univ. of Sydney, AUSTRALIA Plasma Rosearch Leb. Australian Nat Univ. AUSTRalia Prof. I.R. Jones. Flinders Univ. AUSTRALIA Prot. E. Cap. Inst for Theoredcal Physics. AUSTRIA Prot. M. Heindler, Instiut tor Theoretische Physik, AUSTRIA Prof. M. Gooscons, Astronomisch Instuur BELGIUM Ecole Royele Mititure. Leb. de Pry. Plaemas. BELGIUM Commission-Europeen. DG. XII-Fusion Prog.. BELGIUM Prof. R. Bowoqud. Rijksummoribat Gent BELGIUM Dr. P.H. Sakenaks, Instioto Fisica. BRUZIL


Documents Oritos. Alomic Energy of Cenada LU., CANADA Dr. MP. Bectynekx, MPB Tectinotogies, Inc.. CANADA Dr. H.M. Skeregard. Univ. of Seckatchowen, CANADA Prol. J. Toichmam, Unn. of Montreal, CANADA Prot. S.R. Sromivasen. Univ. of Celgary, CANADA Prof. T.W. Johnston, INRS-Energio. CANADA Dr. R. Boton. Contro canacien de husion magnétique, CANADA D. C.R. James. Univ. of Nbort. CANADA Dr. P. Lukte. Komonaketro Univorszita. CZECHOSLOVAKIA The Librarian, Cuham Leocrabry. ENGLANO Lorary, R61, Puthertord Appleton Laboratory. ENGLAND Mr. S.A. Hutctinson, JET Libren, ENGLAND Dr. S.C. Sheme, IJniv. of South Pecific. FIJI ISLANDS P. Manonen, Univ. of Heisinti, FINLAND Prof. M.N. Buseac. Ecoto Polyochnioue. FRANCE C. Nourtat, bu Physiqua des Milioux boniets, FRANCE J. Redal CENCADARACHE - Bat 506. FRANCE Prol. E. Economou. Univ. of Croto. GREECE Ms. C. Finni. Univ. of loamina GREECE. Dr. T. Mue. Acadermy Bibliographic Ser., HONG KONG Propme Libr.y. Hungarian Acadomy of Sai. HUNGARY Dr. B. DasGipa. Saha InsL of Nucber Physics. INDIA Dr. P. Kaw, Inst. for Plasma Reseurch. INDIA O.P. Rocenne; isened inst of Tectinobogy. ISRAEL Librenen, In mamabona Conwer for Thoo Physics. ITALY Miss C. Do Palo, Ascoriazono EURATOMANEA, ITALY Dr. G. Groseo, 1800 in di Fisica del Plasins. ITALY

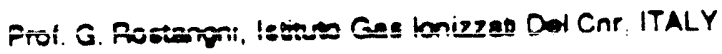
Dr. H. Yumato. Tochiba Pos a Dowd Conter, JAPAN
Prof. I. Kowrakemi. Hiroshima Univ.. JAPAN

Prol. K Nishikewe. Hiroshima Univ.. JAPAN

Orector, Jeaen Atomic Enorgy Rosearch InsL, JAPAN

Prot. S. Ion, Kyustw Univ., JAPAN

Research Into. Cr., Nationa Insul tor Fusion Science, JAPAN

Prof. S. Tenake, Kyobo Univ., JAPAN

Librey, Kyoto Univ., JAPAN

Prot. N. Inowe. Univ. of Tokyo. JAPAN

Secretay, Plesme Section, Electobchnical Lab.. JAPAN

S. Mori, Technical Aovieor, LAERI, JAPAN

D. O. Mirerei, Kunnemoto inst of Technology. JAPAN

J. Hyoon Sook, Kores Abomic Enorgy Posearch Inst, KOREA O.1. Chai, The Koren Adv. Inse of Sa. \& Tech., KOREA

Prot. B.S. Liby. Univ. of Waikato. NEW ZEALAND Inat of Phreica, Chinew Acad Sa PEOPLE'S REP OF CHINA Librey, Inst of Plasma Physics, PEOPLES REP. OF CHINA Tinghue Univ. Library. PEOPLE'S REPUBLIC OF CHINA

Z Li, S.W. Inst Phycia, PEOPLE'S REPUBUC OF CHINA Prof. J.A.C. Cobred, Instuto Superior Teanioo, PORTUGAL Dr. O. Potrus. Al I CUZA Univ., ROMUNIA Dr. J. Villion. Fusion Studies. AEC. S. AFRICA Prot. M.A. Helberg, Univ. of Nam, S. AFRICA Prot. D.E. Km, Pohang Inst. of 30. \& Tech., SO. KOAEA Prot. C.I.E.MA.T. Fusion Division Library. SPAIN Dr. L STomflo, Univ. ." 'MEA, SWEDEN Lorey, Royd Inst of Tectnology, SWEDEN Prot. H. Wihatmeon, Chaimors Univ of Tech. SWEDEN Conte Phys. Des Plasmas, Ecole Potroct, SWITERLAND Bibliotheak. Inst. Voor Plasma-Fysica, THE NETHERLANDS Asel Prot. Dr. S. Covir. Midde East Tech. UnN., TURKEY Dr. VA. Gukhikh, Sa. Ros. Inst Eloctoohys I Appararus, USSR Dr. D.D. Ryubr. Siborian Brench of Academy of Sa., USSR Dr. GA. Eleeer. I.V. Kurchabov Inst. USSR Lbrevien. The Uks. SSR Academy of Scionces. USSA Dr. LM. Kovizinnykh, Inst. of Genera Physucs. USSR Komtorectungeandege GmbH, Zentrabibtiothek, W. GERMANY Bidiouok, inst. Fir Plasmatorschung. W GERMANY Prof. K Schinder. Rutr-Universuti Bochum, W GERMANY D. F. Wagner, (ASOEX), Max-Pland-Instoul, W. GERMANY Litrenen, Max-Plenck-Instudr, W. GERMANY Prot. R.K Jenow. Inst of Prysucs, YUgOSLAVIA 


